\title{
THE MIRROR OF SCIENCE
}

\section{Marek Biernacki, Antoni Smoluk}

\begin{abstract}
Mathematics is a family of theories; there is an interpretation of every science of the physical world in the corresponding mathematical structure. Hence, mathematics is the mirror of science in its entirety.
\end{abstract}

Keywords: mathematics, science, faith.

JEL Classification: C00

DOI: $10.15611 / \mathrm{me} .2018 .14 .01$

\section{Introduction}

Science is mirrored in mathematics, which reflects the whole science. What is mathematics? Mathematics is the science of mathematical structures. It examines relational systems. A mathematical structure is a relational system. We live in a world of relations. A community is defined by the distinguished relations occurring between its members. A crowd is a group pursuing a common goal and guided by a specific logic; this whole could have a goal that is contradictory to the self-esteem of individuals. Gatherings have been little explored. However, every assemblage is tied by a specific relation, has its own sense of individuality; in short, it is a distinguished whole that differs from a set of people. Thus, there is a relation of kinship, neighbourhood, friendship, marriage, etc. Everything is a relation. Systems of relations are mathematical structures. Preferences - which constitute the basis of economics - measure the quality. These are extremely simple relations. Namely, they satisfy only two conditions: firstly, they are transitive, and secondly, they are reflexive. Any two or multi-argument relation is a set. The elements of the set are ordered finite sequences [Biernacki, Smoluk 2018].

The aim of science is, firstly, the cognition of the world, secondly, the improvement of human beings, and thirdly, formal perfection. Hence, science

\footnotetext{
Marek Biernacki

Wrocław University of Economics

e-mail: marek.biernacki@ue.wroc.pl

ORCID: 0000-0002-7738-2065
}

\author{
Antoni Smoluk \\ Wrocław University of Economics \\ e-mail: math@ue.wroc.pl \\ ORCID: 0000-0002-9990-037X
}


is a utilitarian prognostic knowledge, which is also ethical and aesthetic. The Pythagoreans put the main emphasis on the ethical side of science. They perceived science as a type of a prayer bringing humans being closer to God. Steinhaus's tombstone is inscribed with the following words: "There is mathematics mediating between spirit and matter". This mediation is presumably an isomorphism. The spiritual and the material worlds are identical in structure, yet completely different in nature. Is knowledge possible without faith? Science seeks the laws of nature so there is no science without the faith that the laws exist. Faith is more than cognition. There is no knowledge without faith. Deep faith makes the developed theories a reality. In His teachings, Jesus called for self-perfection since God is a perfect being. Gospels often speak of faith which makes anything possible. Perfection and faith is the essence of any knowledge.

The notion of commensurable segments was introduced by the Pythagoreans. The practical significance of this notion is negligible. Any two segments always have a common measure. It is only in geometry operating with ideal objects that the side of the square and its diagonal are incommensurable. We do not know whether a day is commensurable to a month and a month to a year. A good calendar exists regardless of the answer to this question. Ideal objects are a domain of science while applications operate with merely approximate values. Hence, faith is first and science is a result of faith. Scientific knowledge is necessary, it is a kind of prophecy. It is worth mentioning here that the notion of commensurability is a linear dependence of vectors in the space of the infinite dimension of real numbers $\mathrm{R}$ over the body of rational numbers Q. Science idealises. Scientific beings have only distant equivalents in nature. Intuitive connectivity is an arc of connectivity: any two points in space can be connected with an arc. Generally, connectivity means the impossibility to decompose space into two non-empty, disjointed and open sets. Naturally, there are connected spaces, but they are not connected by means of an arc. Science verifies intuition. A pseudo-arc is an example of a connected space, which, however, is not connected by an arc. It is a line being a union of a closed segment with the ends $(0,-1),(0,1)$ and the function

$$
f(x)=\sin (1 / x) \text { dla } 0<x \leq \pi / 2 .
$$

This type of extreme structures may be useful in applied sciences. They serve as models of real objects. 


\section{Isomorphisms and models}

At a seminar organised under the auspices of John Paul II in Castel Gandolfo, Krzysztof Maurin discussed group representation. The motto of his speech, in Greek, taken from St. John's Gospel, was read out by the Pope. For Maurin, an abstract group is WORD - LOGOS, and the group of linear transformations, which is isomorphic to it, is FLESH. Jan Mozrzymas perceived symmetry as the principal rule of the Universe. The notion of symmetry is focused in the transformation group. The world is governed by symmetry. He later generalised this definition - he adopted a semigroup as the foundation of the outer space. This extension means irreversibility of certain states of nature. It is worth mentioning that Mozrzymas's symmetry principle has permeated the whole of Wrocław's intellectual environment. The transformation group bears time since time is a change, a movement. Perfection means a substance clothed in relevant and mnemonic symbolism and a beautiful, educated language of expression. Every scientific work contributes knowledge and is a piece of art - a literary work at the same time [Smoluk 2014]. Mathematics is a science of the physical world in an abstract form. Abstraction is an aspiration for perfection: the maximum simplicity and generality. The forefront of science is always in the half-shadow zone. At first, we are still far from perfection; we grope for the unknown regularities. The laws of nature have a mathematical form - the regularities governing the world cannot be expressed otherwise [Malawski 2016]. If we speak of the dependence of price on supply, we normally express this relation with words: when a price increases, supply increases, and when a price decreases, supply also decreases. These values are bound by a functional dependency and the function is monotonic. Although we do not know this function, we distinguish the whole class of objects when speaking of its monotonicity. Depending on the need, one of the functions will be an appropriate prognostic tool. This is because the most important aim of cognition is prognoses. The notion of monotonicity can be recognised as a law of economics. In the half-shadow zone, where cognition is partial, mathematical models prove highly useful. These are simplified formal descriptions of the physical or social reality, which permit a certain insight into the nature of a phenomenon; a model is a mathematical experiment. Various formal structures are tested in physical observations. The models, although still far from the laws of science, are useful if they contribute the knowledge which specialists in a given field have not had before. It is at the 
same time the simplest evaluation of all applications of mathematics. A mathematical tool is good if and only if it helps understand something, if it brings a new light. If mathematics obscures the examination of the reality, it is a parascientific activity rather than applications of mathematics. Naturally, there are always mistakes and the right model emerges only after several trials. As science progresses and we leave the land of cognitive half-shadow behind, we enter the area of knowledge of the world. Here, the laws of nature are already discovered and, as always, they assume the form of specific relations - sets. Cognition is a continuous process engaging many generations. Changes of paradigms in science do not contradict the continuity of scientific progress. Paradigms create new models, provide a different view, expand semantics, yet do not refute either the beauty or the values which used to apply. The Ulam principle also needs to be remembered. A mathematician will do it better. This law means that out of two persons equally prepared to perform an action, the one who will perform it quicker and better is the one who follows mathematical rules, who is a mathematician by education. Mathematics is first of all the organisation and rationalisation of activities.

The essence of mathematics is isomorphism. Isomorphism identifies various mathematical structures and combines various fields of science where the same laws apply under different names. The content and nature of relations and objects can be different and is different at times, yet the structures are the same. A beautiful and extremely important example of isomorphism is the school-like logarithmic function and its inverse - the exponential function. Obviously, we are speaking here of isomorphism of an additive group of real numbers $\mathrm{R}$ to the multiplicative group of strongly positive real numbers $\mathrm{R}_{+}{ }^{*}$. The labour-intensive multiplication is replaced by an easy addition. This isomorphism means that arithmetic sequences and geometric sequences are the same. A mathematician does not distinguish a geometric sequence from an arithmetic sequence. Banks, however, do distinguish the sequences as it is an enrichment of the language. Arithmetic sequences are simple interest. Simple interest is linear capital growth. Geometric sequences are compound interest in banks. In this case the capital grows exponentially. For a mathematician, linear growth and exponential growth are the same as these are isomorphic notions. For banks and investors these are different matters. Musical tones are defined as a geometric sequence but, according to what was said above, they can be seen as an arithmetic sequence. It also worth shedding a slightly different light on the nature of natural numbers. Does zero exist and is zero a natural number? What is the nature of zero? At times, a set of natural 
numbers begins with zero and at other times - with one. If natural numbers are defined as cardinal numbers of finite sets, the objects are categories. From the logical point of view, zero is the smallest natural number because it is the power of an empty set. The existence of an empty set is also difficult to accept. If an empty set is an empty bag, an empty room, or an empty wardrobe, why would all empty sets be equal? They are not identical objects - a mathematical equality is equivalence. Zero does not exist in nature although there are empty places in nature which are symbolically marked with zero. Numbers are marked by stones and no stone symbolises zero on the abacus. There is undoubtedly the number one in nature since it is a symbol of the origin of all things. All other numbers originate from one. This is perfectly captured in the system of Peano axioms. In this system of axioms, the original notion is the set $\mathrm{N}^{*}$ of natural numbers, the distinguished number 1 , and the function seq called a sequence or a successor. Number 1 is defined as an element which is not a successor of any other number, and every other element $\mathrm{N}^{*}$ is a successor of exactly one number. These axioms mean that the function sequence is multivalued and covers the whole set $\mathrm{N}^{*}$ save for the first element, which is not a successor of any number. This function increases every number by a unit in order to reach the successive number. Peano axioms contain the theorem on the existence of an infinite set since the function sequence is multivalued and maps the whole set of natural numbers onto its part. Hence

$$
N^{*}=\{1,(1+1),(1+1)+1,((1+1)+1)+1, \ldots\}=\{1,2,3, \ldots\} .
$$

Number 2 , by definition, is the sequence $1,2=\operatorname{seq}(1) ; 3=\operatorname{seq}(2)=$ $=(1+1)+1$, etc. The axioms in Peano' system represent natural numbers as partitions; every natural number originates from the unity through successive additions. In his axioms, Peano included a children's game consisting in putting successive lines or matches in rows. Natural numbers excluding zero create a multiplicative semigroup. The nature of this semigroup is very simple. The semigroup is isomorphic to the semigroup of words in the infinite alphabet. In the semigroup of words in any alphabet, the operation called multiplication consists in adding words; a unity is the empty word marked with the symbol 1. If there are two words $s=a b$ and $t=a b b$, then $s t=a b a b b$, while $t s=a b b a b$. A set of natural numbers with the unity $\mathrm{N}^{*}$ is such a semigroup. One is identical to an empty word, whereas every other number is a word formed with prime numbers - a partition of the number into the product of prime factors. Four is the word twotwo, six is the word twothree and the word threetwo, twelve is the word (twotwo)three, etc. Certainly, the semigroup of natural numbers is commutative. Multiplication of natural numbers comes 
down to a simple noting of words next to each other with no operations. The number twelve 223 multiplied by the number eighteen 233 is the number $(223)(233)=222333$. The alphabet in this semigroup is the set of prime numbers. It should be noted here that a semigroup is a set with one associative operation and an identity element. The semigroup $\mathrm{N}^{*}$ is another model of natural numbers excluding zero. The school set of natural numbers excluding zero with an ordinary multiplication operation is a different model. This semigroup is isomorphic to the family of finite sequences of natural numbers. A sequence where almost all the words are zeros is called a finite sequence. Thus, finite sequences of natural numbers form an additive semigroup; the identity element is a sequence made up of zeros only and marked with 0 . Sequences with only one word other than zero, which is one, are assigned to prime numbers, which are letters in the semigroup of words. Respective sequences: $2=(1,0,0, \ldots), 3=(0,1,0,0, \ldots), 5=(0,0,1,0,0, \ldots)$, etc. are assigned to successive prime numbers: $2,3,5, \ldots$ Linear combinations of sequences representing prime numbers are assigned to composite numbers. Hence:

$$
6=(1,1,0,0, \ldots) ; 30=(1,1,1,0,0, \ldots) ; 64=(6,0,0, \ldots) .
$$

Prime numbers form a kind of a base in the set of integers. In this model, the multiplication of natural numbers was reduced to adding finite sequences. If $X$ is a semigroup, a part of the semigroup $\mathrm{N}^{*}$ and every prime number is an element of set $X$, then $X=\mathrm{N}^{*}$. This simple theorem shows again that the semigroup $\mathrm{N}^{*}$ is generated by the set of prime numbers. Let $\mathrm{M}$ mean a family of all finite sequences with integer values. The set $\mathrm{M}$ is a module over the ring $\mathrm{Z}$ of integers. Sequences can be multiplied by integers and added simply with the use of coordinates. An additive group such as the module M, is isomorphic to the multiplicative group $\mathrm{Q}^{*}+$ of strongly positive rational numbers. The multiplication of fractions means here the addition of sequences, and the reciprocal of a rational number is a simple change of signs in a sequence. Hence, if $2=(1,0,0, \ldots)$, then: $1 / 2=(-1,0,0, \ldots), 2 / 3=(1,-1,0,0, \ldots)$, whereas $7 / 15=(0,-1,-1,1,0,0, \ldots)$. As can be noticed, every model expands our knowledge and enriches semantics; the structure is the same - all four models are isomorphic. The following conclusion can be drawn: abstraction simplifies and unites distant branches of knowledge. It is only models that have practical value. An interpretation of a theory is a model; models are at the same time a confirmation of the validity of a theory. The more models, the better, the greater benefit from a good theory. Good theories contribute a lot to cognition and have an enormous practical significance. 
Axiomatic theories, apart from the cognitive value, are characterised by logical beauty. Axioms have their counterparts in the examined reality; there is no science without this relation. The basis axiom of science in its entirety, and mathematics in particular, is the axiom speaking about the existence of an infinite set. What is infinity? Children were asked about the existence of infinity. For children, it is an existing being. Two thirds of the respondents believed that there could be several infinities, and one third held the opinion that there was only one infinity. Most likely the children who thought that there were many infinities meant a potential infinity, while others thought of an absolute infinity. How are these two notions different? Potential infinity is in fact finiteness. It is not argued that there are infinite sets: all sets are finite but there is no biggest set among finite ones. There is no highest number among natural numbers. For the children, continuous production was equal to an infinite stream of goods. The children did not attempt to specify actual infinity. Actual infinity means a set equivalent, in the sense of equipotency, to its proper part. Elimination of a finite number of elements from an infinite set does not diminish the infinity. The axiom of infinity argues that there is an infinite set. This axiom cannot be verified directly. It remains unknown whether infinite sets exist or not. The adoption of the axiom of infinity does not, however, lead to a contradiction; in fact, numerous applications of science confirm its validity, they are an indirect confirmation of the relevance of this axiom. All principles on which contemporary science relies, including the principle of symmetry, stem from the axiom of infinity. The axiom of infinity is a theorem on an ideal world which is incognisable. The whole of contemporary science, which is underlain by numeral systems and vectors, results from this axiom. For an economist, a vector is a basket of goods or a system of prices, while for a physicist - a force. The principle of a numerical description of the states of nature provides that all is a vector in a properly selected system of units. Every state of nature can be described with a sequence of numbers. The principle of a numerical description of the states of nature is a research attitude reducing cognition to measurements rather than a law of nature. The axiom of infinity implies continuity; continuity is a great facilitation and simplification. Continuous functions and the differential calculus are the basis of contemporary science. The only law of nature, a principle that competes with Mozrzymas's symmetry, is the law of equilibrium. The world is in a state of equilibrium. The forces of action which push forward and the forces of reaction which suppress this impulse, the inhibiting forces, neutralise each other. The sum of the forces acting in each point is 
zero: the real world is in equilibrium with the virtual world. Equilibrium is symmetry and symmetry is equilibrium. Organisms exist only in equilibrium. Continuous movement is dynamic equilibrium. The Universe strives for the absolute state of equilibrium. What this state will be remains unknown. Perhaps it will be the starting point of a new big bang.

The world is not governed by chaos. The additive group of the remainders of modulo 2 is isomorphic to the multiplicative group $\{1,-1\}$ of two orientations of the world: left and right. Dirichlet's pigeonhole principle is a simple method of proving theorems. If there are more items than pigeonholes, then there are at least two items in one of the pigeonholes. Hence, if there are three natural numbers, then at least two of them have the same parity - they leave the same remainder when divided by two. A set of matrices with determinants other than zero is not a typologically connected space. Such a space is divided into two homeomorphic connected subsets; one subset is matrices with a positive determinant, that is matrices orienting the space differently than those with negative determinants. One orientation is called right and the other - left. If considering a point of the left part of the space and a point of the right part, then there is no line in the set of non-singular matrices connecting the two points. It is impossible to pass from the right world to the left one in a continuous manner. On the way, one must stop by hell, that is pass the singular matrix with the determinant zero. What arises from the pigeonhole principle is a simple theorem on prime numbers and arithmetic sequences. If $r$ is a natural number other than zero, there is an arithmetic sequence with the difference $r$, a certain sub-sequence of which contains all words being prime numbers. Such an arithmetic sequence exists for every number $r$ other than zero: regardless of the difference of the sequence, there is always an arithmetic sequence that encounters an infinite set of prime numbers. This theorem is a consequence of Dirichlet's principle. The additive group $Z_{r}$ of the remainders of modulo $r$ contains precisely $r$ elements; the elements are classes of integers of equal moduli $r$. The classes can be treated as arithmetic sequences with the same difference $r$ but a different initial word. Since there are infinitely many prime numbers and there are only $r$ classes in the group $Z_{r}$, one of the classes contains infinitely many prime numbers; naturally, there can be a few such classes. Let $a$ mean a sequence of words belonging to the semigroup $\{1,-1\}$, let $s$ remain a sequence of partial sums of the sequence $a$, that is

$$
s=\left(a_{0}, a_{0}+a_{1}, a_{0}+a_{1}+a_{2}, \ldots\right) \text {, where } a=\left(a_{0}, a_{1}, \ldots\right) .
$$


If one means a step right and minus one - a step left, then the sequence $a$ can be called a random walk. If we are in the zero point in the initial period, then questions arise. Is the sequence of partial sums of sequence $b$ limited for each arithmetic sub-sequence $b$ of sequence $a$ ? Do the sums of words of sequence $b$ fall into a certain range of integers $[-n, n]$ ? Supposedly, such a sequence $a$ does not exist. Let us leave this problem open for the reader's amusement. Such research problems, although they can have a practical significance, are considered more as curiosities and logical entertainments.

The probability theory is a science of various definitions. It is uncertain whether there are incidental, random events. There is full determinism in nature. The calculus of probability can be treated as a school teaching on percentages. For a mathematician, it is an axiomatic theory - a special branch of the theory of measure and integration. A normalised measure is identified with probability as these are isomorphic structures. Probability can also be viewed from the geometric perspective. For a geometrician, convex combinations are identical to measures. A triangle is a family of all probabilities assuming only three values, that is a set of ordered threes of nonnegative numbers the sum of which is one, hence every point of the triangle is a certain distribution. Probability is also convexity. Both views on the probability theory are useful and shed different lights on this practically - significant theory. It is difficult to calculate multidimensional integrals without Monte Carlo methods. At the same time it is worth stressing that it is people that bring uncertainty into social life. Presumably, it is only our free will that generates uncertainty in the world we live in. Convex combinations of vectors play a crucial role in the whole analysis. For an ordinary student, probability is a mere percentage: $50 \%$ of the cards are red. The famous work by Nicolas Bourbaki Éléments de mathématique does not mention the calculus of probability. This absence is a screaming silence.

Mathematical equalities are not philosophical identity; they are a relation of equivalence. Equivalence is a preference which, apart from being transitive and reflexive, is also symmetrical. A quarter added to a quarter is a half but every child is perfectly aware that two quarters of an apple are not the same as half an apple. Polyhedra can be identified in terms of volume. Any two polyhedra are equivalent when their volumes are the same. There is a geometrically important relation of equivalence introduced to the family of polyhedra: two polyhedra are equivalent when one of them can be cut by planes into an infinite number of pieces which can be used to assemble the other one. The third famous Hilbert problem is the question of whether any 
two polyhedra of the same volume are equivalent in terms of cross-sections. The answer to this question is negative. The cognitive value of this problem is purely geometric. It is, however, a perfect example of the beautiful relation of equivalence in the set of polyhedra.

The linear space $E$ is called a tensor product of the space $E_{1}, \ldots, E_{k}$ and is marked with the symbol $E_{1} \otimes \ldots \otimes E_{k}$ if there is a universal $k$-linear transformation $\psi$ from the Cartesian product $E_{1} \times \ldots \times E_{k}$ in the space $E$ where, for each linear space $H$ and a $k$-linear map $A$ of the product $E_{1} \times \ldots \times E_{k}$ onto the space $H$, there is an exactly one linear map B of the tensor product $E$ onto $H$ such that

$$
\mathrm{A}=\mathrm{B} \circ \psi .
$$

The tensor product identifies the $k$-linear operator $A$ with the linear operation $B$. The linear operation is a scalar product. The bilinearity of the scalar product has been adopted to the extent that no one perceives this operation as linear. In the linear space $R^{n}$, a scalar product is expressed with the formula $\boldsymbol{x} \boldsymbol{y}=x_{1} y_{1}+\ldots+x_{n} y_{n}$, where $\boldsymbol{x}=\left(x_{1}, \ldots, x_{n}\right), \boldsymbol{y}=\left(y_{1}, \ldots, y_{n}\right)$. Generally, a scalar product can be preset with any positive definite symmetric matrix. A matrix determines the automorphism of the space $R^{n}$. Hence, the scalar product can be viewed from the perspective of this automorphism. The Pythagorean theorem can be represented in a linear space with a scalar product. If vectors $\boldsymbol{a}$ and $\boldsymbol{b}$ are orthogonal, that is $\boldsymbol{a} \boldsymbol{b}=0$, then $(\boldsymbol{a}+\boldsymbol{b})^{2}=\boldsymbol{a}^{2}+\boldsymbol{b}^{2}$; and there is a square of the sum of the vectors on the left and the sum of the numbers on the right. Although it is not directly visible, an isomorphism identifying bilinear forms with matrices is hidden in the Pythagorean theorem.

The foundation of European and global science is logic. Is there any logic other than the one presented by Aristotle in a finite form? It is possible. Mathematics and the entire science are governed by the law of detachment. If the sentence $p=>q$ is true and the predecessor of this implication is also true, then the successor $q$ must be also true. Perhaps there are tribes using different rules of reasoning than our European ones. There is no mathematics as a separate discipline; there is only one science. Mathematics is on the top of the pyramid of knowledge. This mathematical peak rests on isomorphisms. Scientific knowledge is uniquely transferrable. Art does not have this attribute. A great misfortune of the contemporary science is its fragmentation into numerous branches which are isolated from each other. The dispersion of contemporary science is a consequence of the struggle for raising funds for research and receiving a teaching load. Theorems of science are reflected in nature: in dead, living and spiritual matters - social life. 
What is truth? Truth is a relation between the compliance of an utterance and the state of nature to which the utterance refers. This is how truth was defined by Aristotle. His logic is bivalent. It distinguishes only two states of affirmative sentences. Hence there is a state of compliance with nature called truth and identified with one; it is a category of true sentences. The symbol zero designates the category of false sentences. Aristotle does not provide for other possibilities. For various reasons, modal and multivalued logics were created. Two additional functors, which function in the colloquial language, are introduced in modal logic: the necessity functor $N$ and the possibility functor $P$. The sentence $p$ is necessary if its negation is impossible: $N p=\left(P\left(p^{\prime}\right)\right)^{\prime}$, where the prime means negation and the equals sign - equivalence. Negation of a possible sentence can be also a possible sentence (Figures 1 and 2).

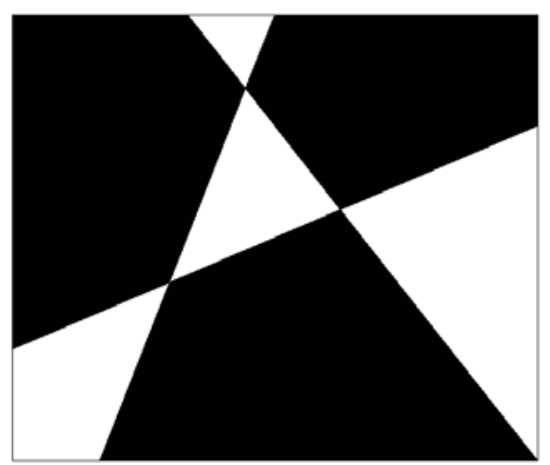

Fig. 1. A possible sentence

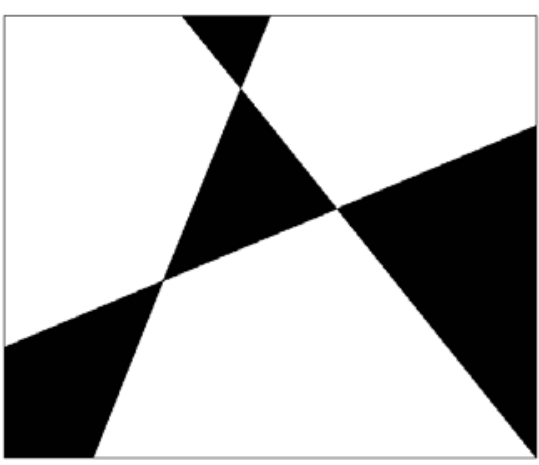

Fig. 2. An also possible sentence

These theories can be useful both in science and in life. In science, they are used when examining the consistency of axioms and in colloquial language they describe states of uncertainty referring mainly to the future. There are multivalued logics or even ones permitting infinitely many states of truth of a sentence. Possible sentences are assigned a half degree of truth and $50 \%$ of falsehood. Hence, a sentence is true if the model indicated by the sentence exists in nature. The sentence $\mathrm{x} R \mathrm{y}$, where $R$ is a certain binary relation, is true if and only if the ordered pair $(\mathrm{x}, \mathrm{y})$ belongs to the set $R$.

Science can be compared to Darwin's theory. Its development is continuous - evolutionary. It is a pyramid: it is soft and blurred at the bottom, it solidifies and crystallises as it rises, and it glows with the diamond of perfect truth, which is known only to God, on the top. God does not command, God does not develop theories and models, God sees. Truth connotes goodness, and goodness connotes truth. Truth cannot be separated from goodness since 
such a divorce means that truth transforms into falsehood and goodness into evil. A majority of theorems are proved without formalism, largely with the use of intuition. Those who see the state of affairs do not need to prove. The mathematisation of theories is not always equivalent to the definition of a semigroup of words. Mathematical theories can be built only after discovering the applicable laws. The laws are recorded in the form of relations; smooth manifolds in finite-dimensional linear spaces - Euclidean spaces often correspond to relations. Mathematisation is a facilitation, a simplification; when idealising, one needs to reject secondary details hampering the examination and leave only what is significant. It is said that patterns speak. Many truths have been inferred using the mindless transformation of words in a semigroup, that is appropriate patterns. A model school type of the problem of words is checking trigonometric and algebraic identities. To prove an identity means to show that one word is equivalent to another one. The problems that are boring to students contain a technique that is characteristic of great science. Everything begins with a word and finishes with a word.

Any square can be assembled with a number of various smaller squares. It has been shown that there need to be at least 21 squares. Obviously, a square cannot be assembled with two different squares, or three or seven. This can be observed without a proof. Such decompositions of a square into various smaller squares are called a perfect cross-section or a perfect partition. Many such partitions have been found and even catalogued. It is undoubtedly a formal, geometric beauty. Even a wardrobe in the form of a square, with doors distributed in accordance with the perfect cross-section of a square into 21 different squares, has been constructed. This furniture reflects the beauty of a mathematical discovery. It is unknown, however, what perfect partition of the square is in nature. Theorems of geometry have natural models; perfect cross-sections of squares also have their equivalents in the physical world. It has been noticed that flows in grids are related to cross-sections of squares into rectangles. Electrical analogies of perfect cross-sections are related to Kirchhoff's laws. It is possible that there are energy zones which in fact model the perfect cross-section of a square, even in our brains. The only relation of the perfect partition of a square with physics can be observed in electrical grids. However the mechanism which would generate the perfect cross-section of a square in a designed large electrical grid is unknown. Such a crosssection can be found automatically when solving many linear homogenous equations. The number of the equations depends on the number of squares into which a given square can be divided. These are equations of equilibrium. 
In fact, the notion of the partition of natural numbers is used. Examining cross-sections of squares is a subsection of the theory of partition of natural numbers. Kirchhoff's laws correspond to equations of equilibrium in electrical grids. Nature conceals its secrets well, one of them being perfect crosssections.

\section{Conclusion}

Proofs are finite sequences of words such that any theorem is a specific semigroup of words. Any mathematical problem can be reduced to the issue of words in an appropriate semigroup. This will be explained using the example of the above-mentioned semigroup of words in the alphabet $\{a, b\}$. Let three axioms: $a a=1, b b=1, a b=b a$ describe the structure of the semigroup. The issue of words in this semigroup comes down to describing the relation of equivalence of words. It can be easily seen that there are only four classes of equivalent words; they are represented by the words: $1, a, b, a b$. All the words where each letter occurs an even number of times are equivalent to unity; the words in which the letter $a$ occurs an odd number of times and the letter $b$ - an even number of times, are equivalent to the word $a$; the words in which $a$ occurs an even number of times and $b$ - an odd number of times, are equivalent to the word $b$, and finally, other words, where $b$ and $b$ occur an odd number of times correspond to the word $a b$. The issue of equivalence of words is easily resolved here. Classes of equivalent words create a multiplicative four-element group with the operation $*: 1^{*} \mathrm{x}=\mathrm{x}, a^{*} a=1, b^{*} b=1$, $a b^{*} a b=1, a^{*} b=b^{*} a=a b, a^{*} a b=b, b^{*} a b=a$. This group is commutative. In general, there is no such a simple algorithm. This four-element group is isomorphic to the multiplicative group of four square matrices being symmetries of the plane: $\{\mathrm{I}, \mathrm{A}, \mathrm{B}, \mathrm{C}\}$, where $\mathrm{I}$ is an identity matrix, $\mathrm{A}$ is a reflection in axis $\mathrm{X}, \mathrm{B}$ is a reflection in axis $\mathrm{Y}$, and $\mathrm{C}$ is central symmetry - a reflection in the beginning of the coordinate system.

$$
I=\left[\begin{array}{ll}
1 & 0 \\
0 & 1
\end{array}\right], A=\left[\begin{array}{rr}
1 & 0 \\
0 & -1
\end{array}\right], B=\left[\begin{array}{rr}
-1 & 0 \\
0 & 1
\end{array}\right], C=-I=\left[\begin{array}{rr}
-1 & 0 \\
0 & -1
\end{array}\right] .
$$

The abstract problem has found its beautiful geometric interpretation: the prophecy has become the reality. Mathematics is the mirror of science, and linear algebra - the science of vectors and linear spaces - is the mirror of mathematics. A linear space can be spread over every set. The elements of the set create the algebraic basis for the space. In practice, the elements of the 
base set are physical units: kg, sec, m, ...; monetary units: PLN, euro, \$, ...; goods units: pieces, $\mathrm{kg}$ of substances, parcels,..., etc. A set of units generates a multiplicative commutative group. Linear algebra spread over the group is a scientific description of a selected fragment of reality. It is the algebra of denominate values. The basic theorem of linear algebra is the parallelogram law. It is a beautiful law of nature. Two forces applied in a point enforce its movement along the diagonal of the parallelogram whose vectors are legs. What arises from the parallelogram law is the Pythagorean theorem and the law of reflection. Isomorphism is the basis of the scientific abstraction, perfection can be found in isomorphism [Smoluk 2017].

\section{Bibliography}

Biernacki M., Smoluk A. (2018). Matematyka i matematyzacja (forthcoming).

Malawski A. (2016). Matematyzacja ekonomii: matematyczność czy matematyzowalność rzeczywistości gospodarczej. [In:] Metaekonomia. Zagadnienia z filozofii ekonomii. M. Gorazda, Ł. Hardt, T. Kwarciński (eds.). Wydawnictwo Copernicus Center Press. Kraków, pp. 131-157.

Smoluk A. (2014). Nauka i sztuka. Didactics of Mathematics. No. 11(15). Wydawnictwo Uniwersytetu Ekonomicznego we Wrocławiu, pp. 85-116.

Smoluk A. (2017). Algebra liniowa. Wydawnictwo Uniwersytetu Ekonomicznego we Wrocławiu. Wrocław. 